\title{
Modeling the extraction of sputtered metal from high power impulse hollow cathode discharges
}

\author{
Mohammad Hasan, Iris Pilch, Daniel Söderström, D. Lundin, Ulf Helmersson and N. \\ Brenning
}

\section{Linköping University Post Print}

\section{Tweet}

N.B.: When citing this work, cite the original article.

Original Publication:

Mohammad Hasan, Iris Pilch, Daniel Söderström, D. Lundin, Ulf Helmersson and N. Brenning, Modeling the extraction of sputtered metal from high power impulse hollow cathode discharges, 2013, Plasma sources science \&amp; technology (Print), (22), 3, 035006. http://dx.doi.org/10.1088/0963-0252/22/3/035006

Copyright: Institute of Physics: Hybrid Open Access http://www.iop.org/ 


\title{
Modeling the extraction of sputtered metal from high power impulse hollow cathode discharges
}

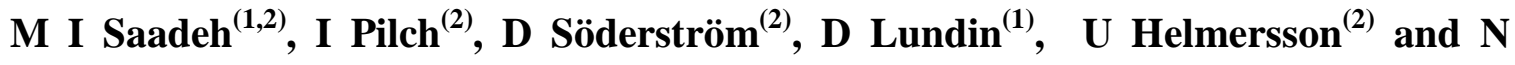 \\ Brenning $^{(1)}$
}

1: Space and Plasma Physics, EES, Royal Institute of Technology (KTH), Stockholm

Sweden

2: Plasma \& Coating Physics Division, IFM-Material Physics, Linköping University, SE581 83, Linköping Sweden

Email: nils.brenning@ee.kth.se

\begin{abstract}
High power impulse hollow cathode sputtering is studied as a means to produce high fluxes of neutral and ionized sputtered metal species. A model is constructed for the understanding and optimization of such discharges. It relates input parameters such as the geometry of the cathode, the electric pulse form and frequency, and the feed gas flow rate and pressure, to the production, ionization, temperature, and extraction of the sputtered species. Examples of processes that can be quantified by use of the model are the internal production of sputtered metal and the degree of its ionization, the speed and efficiency of out-puffing from the hollow cathode associated with the pulses, and the gas back-flow into the hollow cathode between pulses. The use of the model is exemplified with a special case where the aim is the synthesis of nanoparticles in an expansion volume that lies outside the hollow cathode itself. The goals are here a maximum extraction efficiency, and a high degree of ionization of the sputtered metal. It is demonstrated that it is possible to reach a degree of ionization above $85 \%$, and extraction efficiencies of $3 \%$ and $17 \%$ for the neutral and ionized sputtered components, respectively.
\end{abstract}

\section{Introduction:}

Hollow cathode discharges have been in use for a long time, and are operated in wide ranges of parameters depending on the intended application. Some examples are high power hollow cathode arcs [1,2], medium power hollow cathode glow discharges [3], and low power hollow cathodes used for space charge neutralization of the exhausts of plasma ion thrusters [4]. In the present work we combine the hollow cathode geometry with the recently developed technique of high power impulse sputtering, introduced 1999 when Kouznetsov et al. [5] proposed it for magnetron sputtering. For reviews of high 
power impulse magnetron sputtering (HiPIMS) see for instance [6]. In HiPIMS, short high amplitude negative voltage pulses are applied to a sputtering target. The result is a high electron density in front of the target $\left(10^{18}-10^{19} \mathrm{~m}^{-3}[6]\right)$, and a high rate of electron impact ionization of the sputtered species as they pass through this region. By keeping a low duty cycle it is possible to use a momentary power density of up to $10 \mathrm{~kW} \mathrm{~cm}{ }^{-2}$ without damaging the target. Having the sputtered species ionized makes it possible to control its transport by electric and/or magnetic fields. This controllability is very important in customizing the discharge to maximally suit different applications [6]. In this work, we study high power impulse hollow cathode sputtering (HiPIHCS, pronounced "high-picks”) discharges. Two important differences between HiPIMS and HiPIHCS are illustrated in Fig. 1. First, electron confinement in HiPIMS is achieved by magnetic trapping, and in HiPIHCS by electrostatic trapping between the walls of the hollow cathode. Second, in HiPIMS the sputtered species (metal, $M$ ) is geometrically free to escape from the target, while in HiPIHCS extraction is limited to the hollow cathode exit.

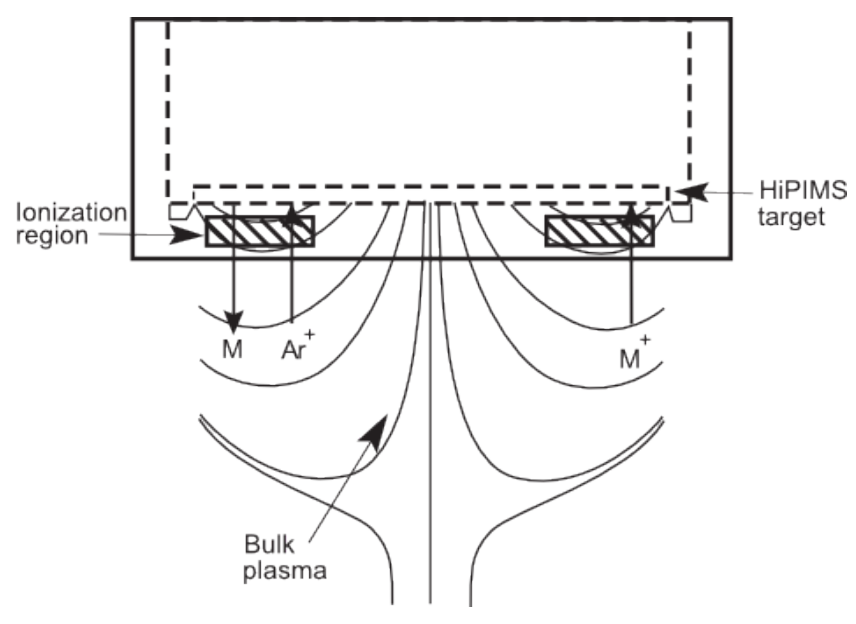

(a)

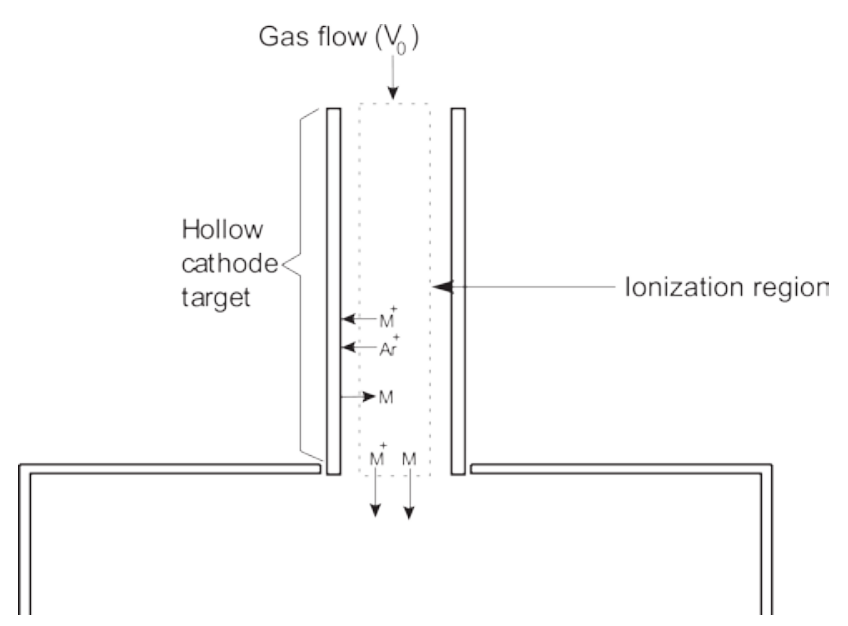

(b)

Figure 1: A comparison between high power impulse magnetron sputtering, HiPIMS, and high power impulse hollow cathode sputtering, HiPIHCS, as sources of sputtered target metal atoms $M$ and ions $M^{+}$. (a) Magnetron geometry with magnetic electron confinement, process gas rarefaction, and geometrically free escape of sputtered material. (b) Hollow cathode geometry with electrostatic electron confinement, process gas heating, and escape of sputtered material through the exit. 
The hollow cathode discharge has two important features. First, being trapped inside the hollow cathode, ions participate in the sputtering process more efficiently than in the more open magnetron discharge geometry, which makes it possible to get a super saturated metal vapor in the hollow cathode discharge. Second, the high plasma density and electron temperature inside the hollow cathode increase the probability for sputtered material to become ionized, which makes hollow cathode discharges efficient sources of highly ionized plasma [7].

The electric field at the exit of the hollow cathode is the key parameter for the extraction of the ionized sputtered species. In a typical hollow cathode direct current discharge there is a limited possibility to control it. In a pulsed discharge there are more degrees of freedom available. Those extra degrees of freedom have a potential of being used to control the discharge characteristics, one of which is the electron pressure gradient at the exit. For cases where the electron pressure gradient term in the Generalized Ohm's law [8] has opposite sign and is larger than the resistive term, a field reversal occurs which is analogous to that sometimes seen in glow discharges between the negative glow and the positive column [9]. The difference between the electron pressure gradient during the pulse-on and the pulse-off times can be large, which affects the strength and can also change the direction of the electric field at the exit.

This paper presents a theoretical model of HiPIHCS discharges, which relates input parameters such as the geometry of the cathode, the electric pulse form and frequency, and the feed gas flow rate and pressure, to the production, ionization, temperature, and extraction of the sputtered species. The use of the model is exemplified with a special case where the aim is the synthesis of nanoparticles in an expansion volume that lies outside the hollow cathode itself, as will be described in the next section. The goals are here a high degree of ionization of the sputtered species and a high degree of extraction. The model is also currently being used, run with a different set of input parameters, for a hollow cathode based discharge for thin films deposition [10] where the optimization goals are different.

The paper is organized as follows. The experiment to be modeled is described in section 2. Section 3 describes the model construction. The modeling results with emphasis on process gas flow, the degree of sputtered species ionization, and the extraction efficiency are given in section 4. Finally, section 5 gives a summary and discussion. Details of the model are given in appendices $\mathrm{A}$ and $\mathrm{B}$. 


\section{The experimental setup}

We here apply the model to the setup for copper nanoparticle synthesis shown in figure 2a, where material is sputtered from a hollow cathode. Nanoparticles are formed in an expansion zone outside the hollow cathode exit (see figure 2a) and collected on substrates. The process gas (argon) is fed at a flow rate of $100 \mathrm{sccm}$ through the hollow cathode. The applied pulse is shown in figure 2b. It has an amplitude of $600 \mathrm{~V}$, a repetition frequency of $500 \mathrm{~Hz}$ and a duty factor of $1.5 \%$ (a pulse duration of 30 $\mu \mathrm{s})$. The gas in the expansion volume is held at a pressure of $p_{0}=133 \mathrm{~Pa}$. The goal is to operate the discharge in a mode that creates a favorable plasma environment for size control and efficient growth of nanoparticles in the expansion volume below the hollow cathode. The desirable environment is described in a patent [11]. There it is shown that a high degree of ionization is beneficial for the growth of nanoparticles because the probability for collection of (positively) charged ions by a (negatively) charged nanoparticle can be orders of magnitude higher than for neutral atoms, which leads to an increase in growth speed [11]. First experiments on size control are reported in [12].

In this application it is desired to maximize both the sputter production inside the hollow cathode and the extraction efficiencies of metal atoms $M$ and ions $M^{+}$at the hollow cathode exit. There is also a need to know how to tailor the subsequent drift and expansion in the expansion volume so that the time developments of electron temperature $T_{\mathrm{e}}$, metal atom density $n_{\mathrm{M}}$, and metal ion density ${n_{\mathrm{M}}}^{+}$can be controlled. The path to achieve these goals can be separated into four stages. First, understanding of the process gas flow patterns associated with the continuous flow and with the pulses; second, modeling the pulse-on internal plasma chemistry in the hollow cathode; third, maximizing the extraction mechanisms; and fourth, controlling the development in the expansion volume. 


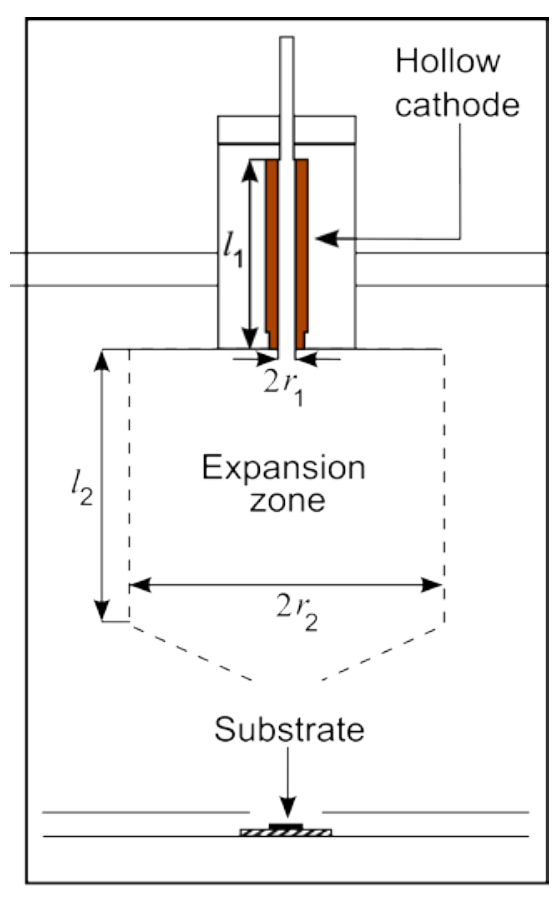

(a)

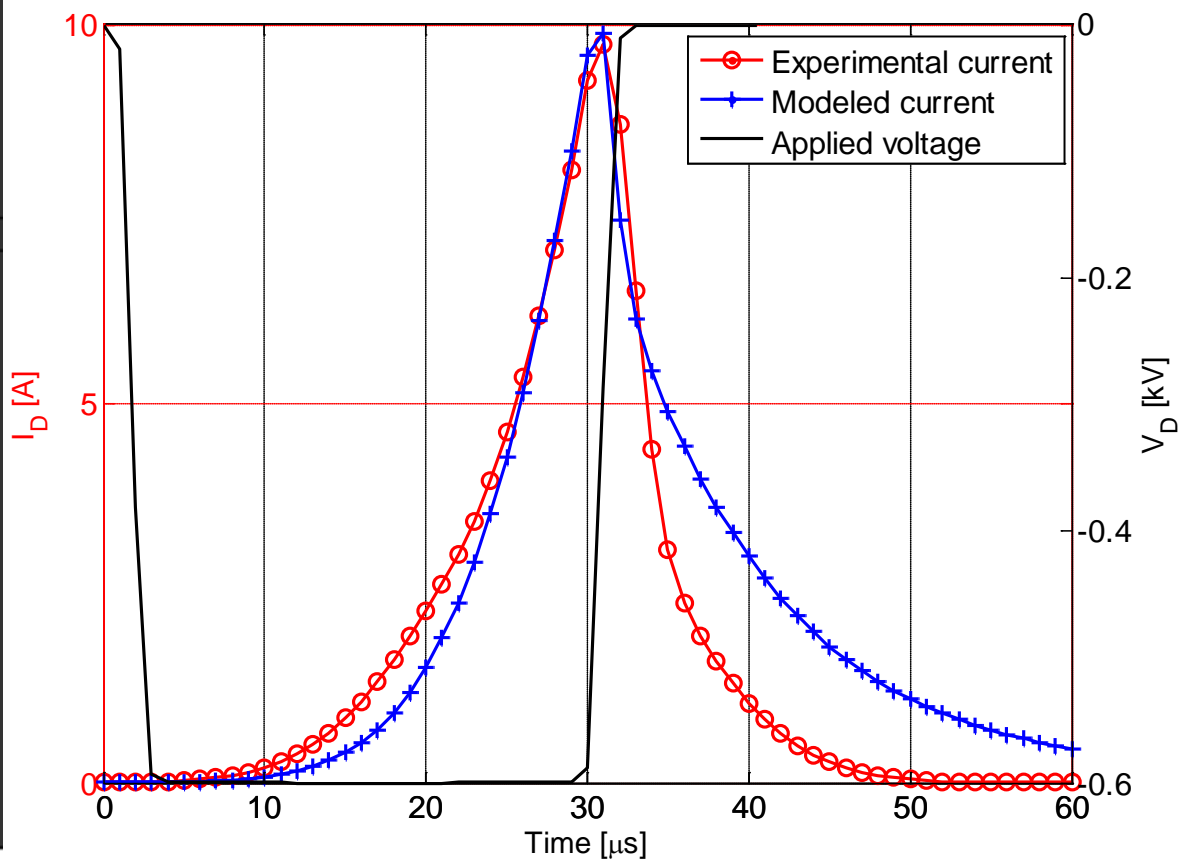

(b)

Figure 2: The experiment. (a) A hollow cathode with the dimensions $l_{1}, r_{1}$ is placed at one end of a grounded cylindrical expansion volume with the dimensions $l_{2}, r_{2}$. Process gas is supplied at the top end at a rate of $100 \mathrm{sccm}$, and the lower end (the exit of the hollow cathode) defines the coordinate $z=0$. The pressure in the expansion volume is kept at $p_{0}=$ $133 \mathrm{~Pa}$. (b) the applied voltage pulse (solid black), the experimental current waveform (circles marked red), and the current calculated by the model (plus marked blue).

\section{Model structure}

The model consists of three rotationally symmetric sub models that are applied to different parts of the process volume and are interlocked as described in the caption of figure 3a. A gas flow model (sub model 1) solves the dynamics of process gas flow, the gas density, and the gas temperature in the whole process volume. A plasma chemistry model (sub model 2) applies only to the hollow cathode, and an extraction model (sub model 3) only to the volume outside of the hollow cathode. The plasma chemistry model has been computed using MATLAB, while the other two sub models have been computed using COMSOL Multiphysics. Although the cathode used in the experiments is made of copper, an aluminum cathode is assumed in the model because of the availability of a reaction data set for aluminum. 


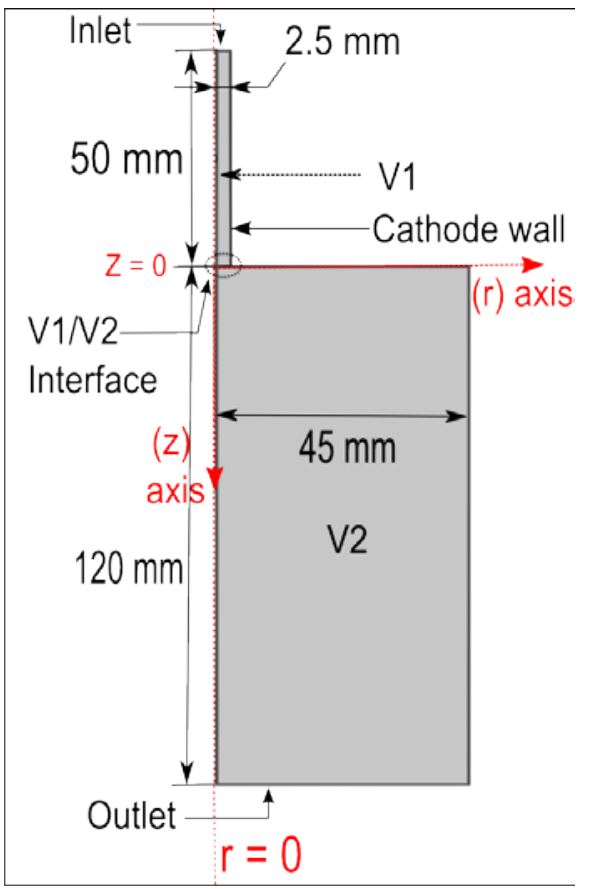

(a)

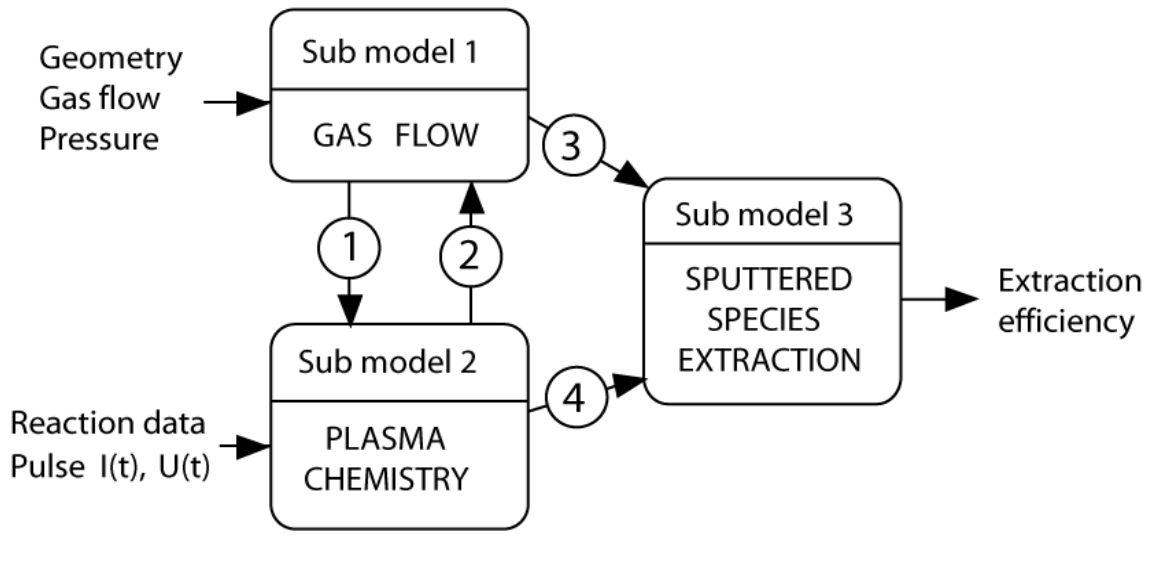

(b)

Figure 3: Overview of the model construction. (a) Geometry of the rotationally (around $r=0$ ) symmetric model showing the hollow cathode volume (V1) and the volume of the expansion zone (V2). The gas flow sub model applies to the whole volume V1 and V2. The plasma chemistry sub model applies only to V1, and the sputtered species extraction sub model only to V2. (b) The interconnections between the three sub models. (1) The density variations of the process gas, along the hollow cathode due to the feed flow, is used to verify the zero dimensional approximation of the plasma chemistry model. (2) The heat influx to the process gas by sputtered atoms, and by energetic recombined process gas ions, is used to calculate the pressure increase during the pulse. (3), (4) The flow speed of the process gas and its temperature at the hollow cathode exit, and the densities $n_{\mathrm{M}}$ and $n_{\mathrm{M}}+$ at the hollow cathode exit, are used to obtain the extraction of the sputtered metal M.

The gas flow sub model is a 2D COMSOL model that solves the fluid flow equations (conservation of mass, momentum and energy) for the process gas. A detailed description of the model and its initial/boundary conditions is given in appendix B. The input consists of the geometry of the hollow cathode and the expansion volume, the pressure in the expansion volume, and the flow rate of the feed gas. The model is first run in steady state case to compute the background flow pattern (the pressure and the velocity before a pulse) which serves as initial conditions for the flow during the pulse. During the pulse, the gas inside the hollow cathode is heated by the flux of the sputtered species and by elastic collisions with electrons, which both are taken from the plasma chemistry sub model. The outputs of the gas flow model are the velocity, the pressure, and the temperature of the gas both in the hollow cathode and in the expansion volume. 
The plasma chemistry sub model follows the collision induced volume chemical reactions inside the hollow cathode, and the particle-wall interactions of sputtering and secondary emission of electrons. The included species are electrons, neutral and singly ionized process gas and sputtered species, and argon atoms excited to the metastable level. The electrons are assumed to have a thermal distribution. The model computes the development in time, during and after a pulse, of the volume-averaged densities and temperatures of these species. The input consists of the hollow cathode geometry, the pulse parameters $I_{D}(t)$ and $V_{D}(t)$ from the experiment, the initial values of the densities and temperatures, the elastic and inelastic cross sections for collisions, and the sputtering yields and secondary emission coefficients for the surface reactions. This model builds on a series of spatially averaged (zero-dimensional) plasma chemistry models developed for sputtering magnetrons [13,14,15]. The model closest to ours is the ionization region model (IRM) of Raadu et al. [15] developed for the ionization region shown in Fig. 1a. The reaction data set for aluminum we use is taken from [15]. The modifications of the plasma chemistry model compared to IRM are summarized in appendix A. Zero dimensional models as this one are attractive because they are easy to implement numerically and yet accurate. The drawbacks are that this type of model does not include the electric potential structures, the electric fields, or the current conduction mechanisms. It also approximates with averages the spatial variations of species densities and temperatures.

In the plasma chemistry sub model, the electron temperature is modeled using the experimental current $I_{D}(t)$ and the applied voltage $V_{D}(t)$ as inputs. The model is made self consistent by varying a fitting parameter $F_{p w r}$, which is defined to represent the portion of applied power delivered to the electrons in the plasma (i.e $F_{p w r}=P_{e} /\left(V_{D} I_{D}\right)$ ). The model solves for $F_{p w r}$ value that minimizes the difference between the experimental current and the modeled current as function of time. This fitting procedure is described in [15]. The agreement between the experimental current and the modeled current is imposed during pulse-on time only since, after pulse-off, the expression that is used to evaluate the modeled current (Equation A.5) is not applicable. The less good current fit after pulse off in Fig. 2b, however, introduces no error in the plasma chemistry since the current then is multiplied with zero voltage, giving the correct zero power.

The extraction sub model treats the fluxes in the expansion volume of the metal and argon ions and the metal atoms: the convection with, and diffusion relative to the argon flow pattern obtained from the gas flow sub model. It is a 2D COMSOL model that solves the 2D drift diffusion equations for the different species. The details of the model are described in appendix B. Since the discharge is collision dominated we assume that all heavy species, $A r^{+}, A r^{m}, M$, and $M^{+}$, have the same temperature as the background argon gas. The boundary condition at the hollow cathode exit area is 
obtained from the time varying densities computed in the plasma chemistry sub model. A summary of the inputs and the outputs of the three sub models and how they are related is given in Table 1.

\begin{tabular}{|c|c|c|}
\hline Sub model & Inputs & Outputs \\
\hline $\begin{array}{l}\text { Gas flow } \\
\text { (sub model 1) }\end{array}$ & $\begin{array}{l}\text { 1. Heating during the pulse. } \\
\text { 2. Geometry of the hollow cathode } \\
\text { and the expansion volume. } \\
\text { 3. Feed gas flow and temperature. }\end{array}$ & $\begin{array}{l}\text { Temperature and velocity of the } \\
\text { background argon gas (i.e. argon } \\
\text { atoms) in the hollow cathode and the } \\
\text { expansion volume. }\end{array}$ \\
\hline $\begin{array}{l}\text { Plasma chemistry } \\
\text { (sub model 2) }\end{array}$ & $\begin{array}{l}\text { 1. Process gas pressure. } \\
\text { 2. Pulse parameters. } \\
\text { 3. Cross sections and the particle - } \\
\text { wall interaction data (see } \\
\text { Appendix A). } \\
\text { 4. Cathode wall temperature. }\end{array}$ & $\begin{array}{l}\text { 1. Spatially averaged densities as } \\
\text { functions of time. } \\
\text { 2. Spatially averaged } \\
\text { temperatures } \\
\text { 3. Fluxes of different species to } \\
\text { walls. }\end{array}$ \\
\hline $\begin{array}{l}\text { Sputtered species extraction } \\
\text { (sub model 3) }\end{array}$ & $\begin{array}{l}\text { 1. Densities of the different species } \\
\text { at the exit. } \\
\text { 2. Temperature and velocity of the } \\
\text { background argon gas in the } \\
\text { expansion volume. }\end{array}$ & $\begin{array}{l}\text { Fluxes of the sputtered species out of } \\
\text { the hollow cathode exit (assuming } \\
\text { ambipolar diffusion for ions). }\end{array}$ \\
\hline
\end{tabular}

Table 1. The input and output parameters of the three sub models.

\section{Model results}

In this section the physical processes are described: first the gas flow during and after a pulse, then the plasma chemistry during and after a pulse, and finally the extraction of plasma and sputtered species out of the hollow cathode exit.

\subsection{The process gas: flow patterns and temperature}

Figure 4a shows the gas flow pattern in steady state, before the pulse is applied. Inside the hollow cathode the steady state velocity varies linearly along the axial axis from $26 \mathrm{~ms}^{-1}$ (averaged over entrance area) to $43 \mathrm{~ms}^{-1}$ (averaged over exit area). The pressure also varies linearly from $180 \mathrm{~Pa}$ at the entrance to $133 \mathrm{~Pa}$ at the exit. Figures $4 \mathrm{~b}$ and 4c show the averaged velocity over the exit area for pulsed operation. Here the gas flow sub model has been solved for $4000 \mu$ s (two pulses) in order to see if the pulses mutually interact. The gas is flowing out of the hollow cathode at all times. It takes $10-$ $20 \mu$ s after pulse-on before the downward velocity through the exit starts to increase, and it reaches a maximum of almost $190 \mathrm{~ms}^{-1}$ at $52 \mu \mathrm{s}$, after the pulse-off. Then the outflow slows down until it, during approximately 250 - $950 \mu \mathrm{s}$, has values lower than the steady state gas flow. This is a tendency towards back-flow, driven by an under-pressure inside the hollow cathode due to cooling of the gas 
after the pulse. The disturbance of the gas flow due to the pulse lasts for about $1000 \mu$ s and, at the used pulse frequency of $500 \mathrm{~Hz}$, there are thus no overlapping effects inside the hollow cathode between consecutive pulses.

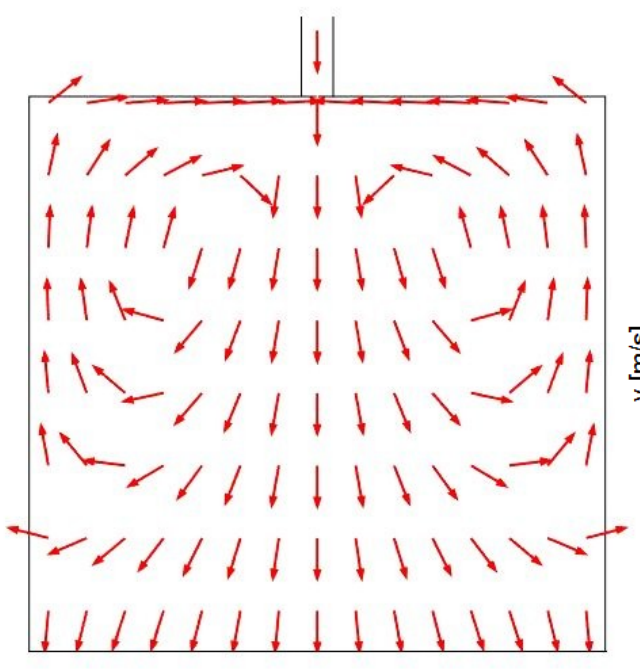

(a)

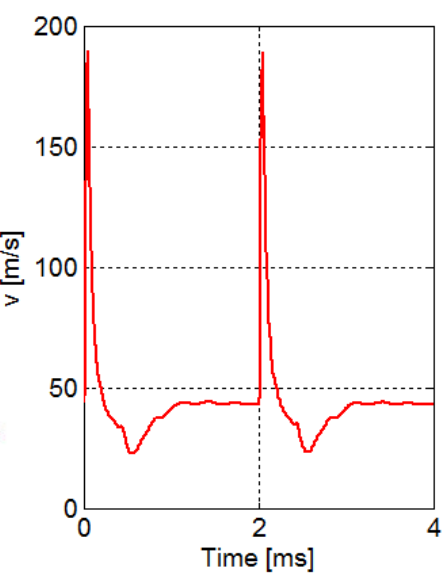

(b)

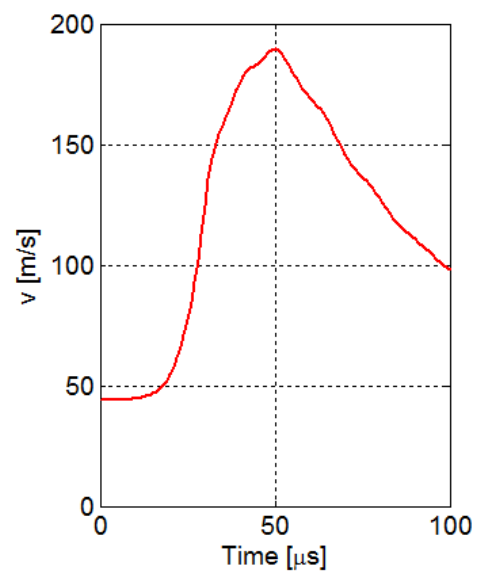

(c)

Figure 4: (a) The steady gas flow pattern shown as a normalized velocity field (i e, every vector has unit magnitude). (b) Downward gas flow speed in pulsed operation, averaged over the exit area of the hollow cathode $(z=0 \mathrm{~mm})$. Pulses are applied during times $0-30$ $\mu$ s and 2000 - $2030 \mu$ s. (c) Same as panel (b) but with an expanded time scale.

Inside the hollow cathode the gas temperature increases rapidly during the $30 \mu$ s long pulse to a maximum of about $1000 \mathrm{~K}$ at the end of the pulse as will be shown below. After the pulse it cools down in about $50 \mu$ s, mainly due to heat exchange with the walls. The time with enhanced gas temperature inside the cathode maintains a pressure gradient along the hollow cathode, and therefore the gas speed continues to increase also after the end of the pulse at $30 \mu$ s as seen in figure 4c. Figure 5 shows the evolution of the gas temperature between the pulses, both inside the hollow cathode and in a part of the expansion volume near the exit. The four sub panels correspond to times $100 \mu \mathrm{s}, 500 \mu \mathrm{s}$, $1000 \mu$ s, and $2000 \mu$ s. Please note that the $100 \mu$ s panel has a different temperature scale than the others. Although the pulse ends at $30 \mu$ s, its effect in the expansion volume starts to appear first around $80 \mu \mathrm{s}$. At that time, a cloud of hot argon gas is blown out of the hollow cathode volume, moves away from the hollow cathode exit and expands longitudinally and radially in the expansion volume. This hot cloud represents a sample of the gas that was inside the hollow cathode volume during the pulse, 
which means that it carries sputtered species along. The center of the cloud has moved away from the hollow cathode about $27 \mathrm{~mm}$ when the next pulse is applied. The velocity field induced by the pulse (not shown here) adds to and perturbs the velocity field of the background flow of figure 4a. Before the time of the next pulse however, the steady flow pattern of figure 4a is restored.
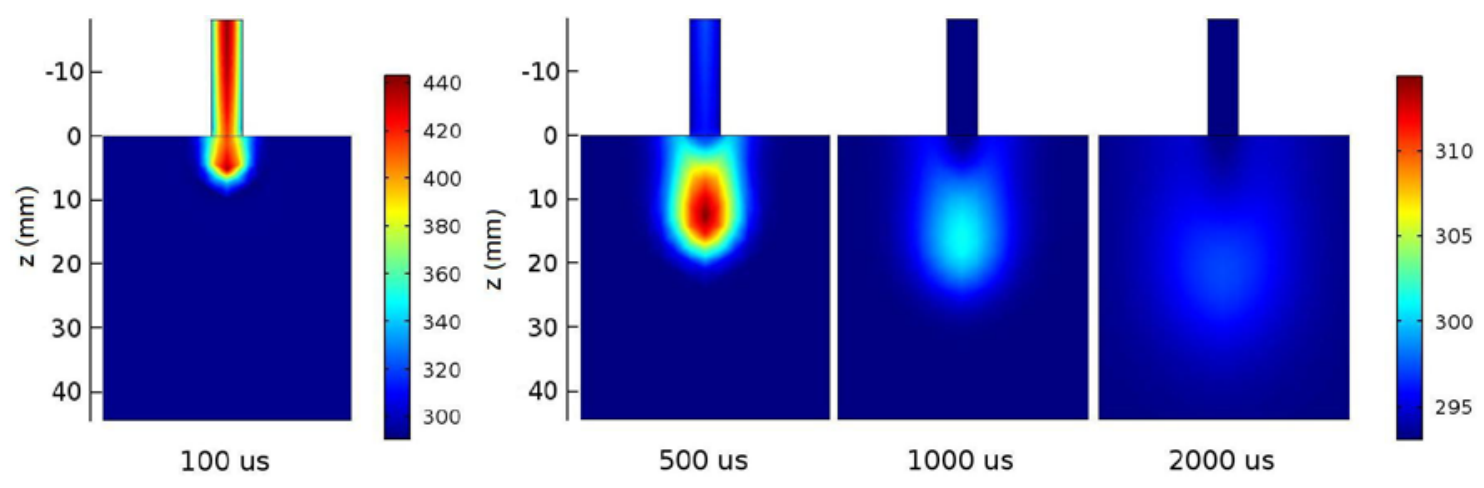

Figure 5: The gas temperature (in Kelvin) in the expansion volume at four different times after pulse start: $100 \mu$ s (please note the different scale in this panel), 500, 1000, and $2000 \mu$ s i.e., at the time of the next pulse.

\subsection{Plasma chemistry inside the hollow cathode}

The plasma chemistry sub model, being zero-dimensional, neglects (1) the pressure drop from $180 \mathrm{~Pa}$ to $133 \mathrm{~Pa}$ along the hollow cathode, (2) the replacement (and loss) of gas in the upper (and lower) ends, and (3) the extra ejection of gas during the last 10-15 $\mu$ s of the pulse (see Fig. 4c). The two latter approximations are motivated by the fact that the feed gas flow during a pulse corresponds only to a flow distance of $43 \mathrm{~m} \mathrm{~s}^{-1} \times 30 \mu \mathrm{s} \approx 1.3 \mathrm{~mm}$ (see figure 4b), which is negligible compared to the length of the hollow cathode; this conclusion is represented by the arrow (1) in the diagram for the sub model interactions in figure 3b. To assess the effect of the pressure variations along the cathode, the plasma chemistry model was solved for three different pressures: $133 \mathrm{~Pa}$ (the pressure at the exit of hollow cathode), $160 \mathrm{~Pa}$ (the pressure in the middle), and $180 \mathrm{~Pa}$ (the pressure at the entrance). The three solutions showed no significant differences in the key modeled parameters. We conclude that the zero-dimensional approximation is valid for the case under investigation.

The plasma chemistry model was solved from the beginning of the pulse until $100 \mu \mathrm{s}$. The value of the fitting parameter $F_{p w r}$ calculated by the model to equate the experimental current to the modeled current (see figure 2b) for this run was 0.25. A sample of the most relevant output parameters is shown in Fig. 6. The electron temperature rises suddenly to $2.7 \mathrm{eV}$ when the pulse is applied, and then 
decreases gradually to $1.8 \mathrm{eV}$. When the pulse is switched off it drops in a few $\mu$ s to around $1 \mathrm{eV}$, and then decreases much slower. The reason for the slow decrease below $1 \mathrm{eV}$ is that, after the pulse, there is a significant population of argon atoms in the metastable level (not shown here). The energy dependencies of electron collisions with atoms in this level are such that above $T_{e} \approx 1 \mathrm{eV}$ energy loss collisions dominate while, for lower temperatures, superelastic (depopulation to the ground state, associated with electron energy gain) become more important. Figure 6b shows the electron density. It starts to rise when the pulse is switched on, peaking at the end of the pulse. When the pulse is switched off it declines with a time constant of about $10-15 \mu \mathrm{s}$. The same description applies to the metal ion density shown in figure 6d. Figure 6c shows the sputtered metal atom density. During the pulse, the sputtering inflow from the walls dominates over the ionization losses $\left(e+M \rightarrow 2 e+M^{+}\right)$. After the pulse, however, the metal atom density decreases faster than the metal ion density because the metal atoms are subject to extra volume losses in the form of charge exchange $\left(A r^{+}+M \rightarrow A r+M^{+}\right)$and Penning ionization ( $A r^{m}+M \rightarrow A r+M^{+}+e$ ) collisions. 

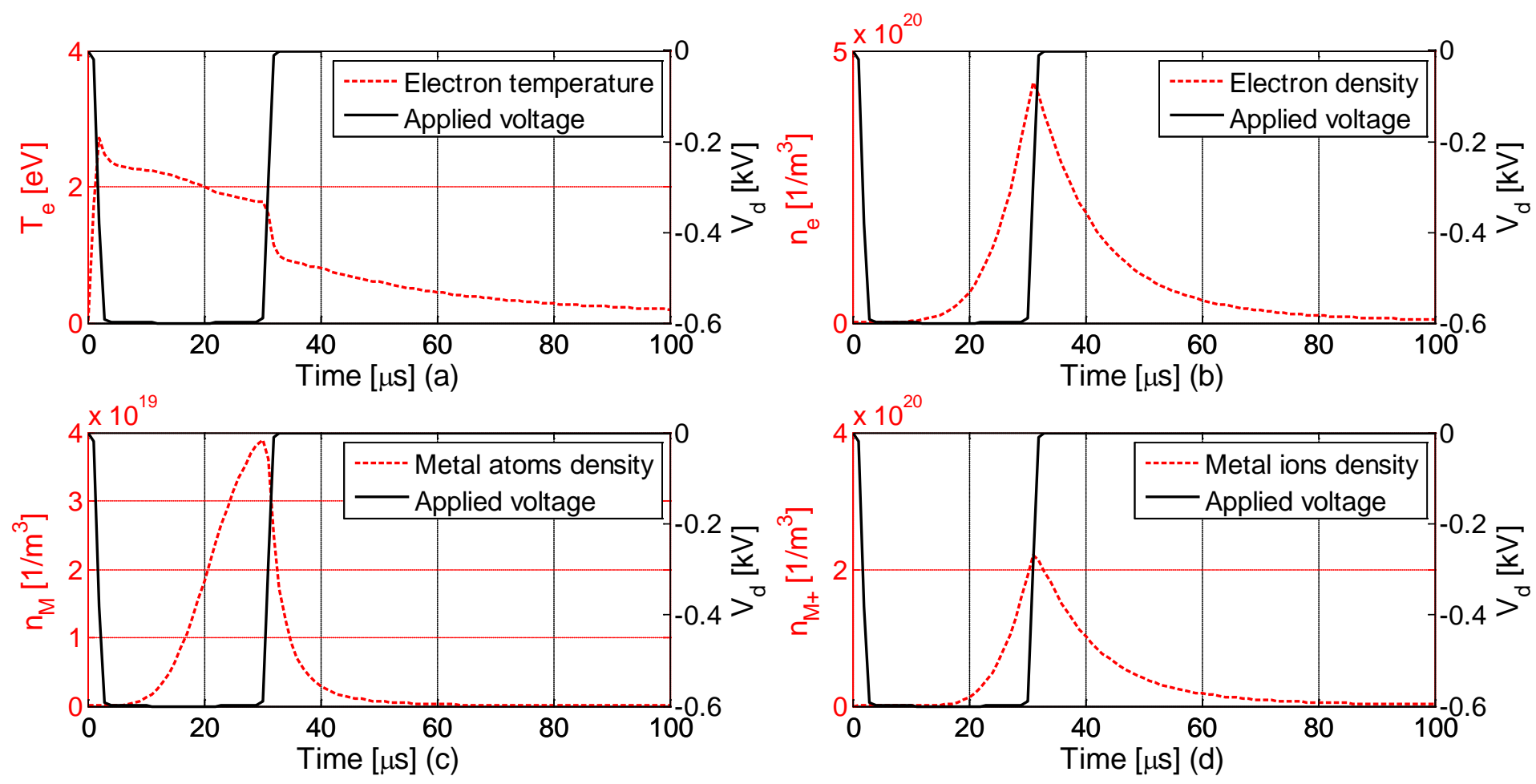

Figure 6: Averaged parameters inside the hollow cathode during and after a pulse, in dashed red (the applied voltage pulse has been inlaid in solid black for reference). (a) The electron temperature. (b) The electron density. (c) The metal atom density. (d) The metal ion density.

The ionization percentage of the sputtered metal species is shown in figure 7, and reaches a value of $85 \%$ at the end of the pulse. It increases further after the voltage is switched off, but here the dominant ionization mechanism changes from electron impact ionization to charge exchange and Penning ionization. Note that when the voltage is switched off, the sputtering rate drops to zero, and no new metal atoms are added to the discharge. 


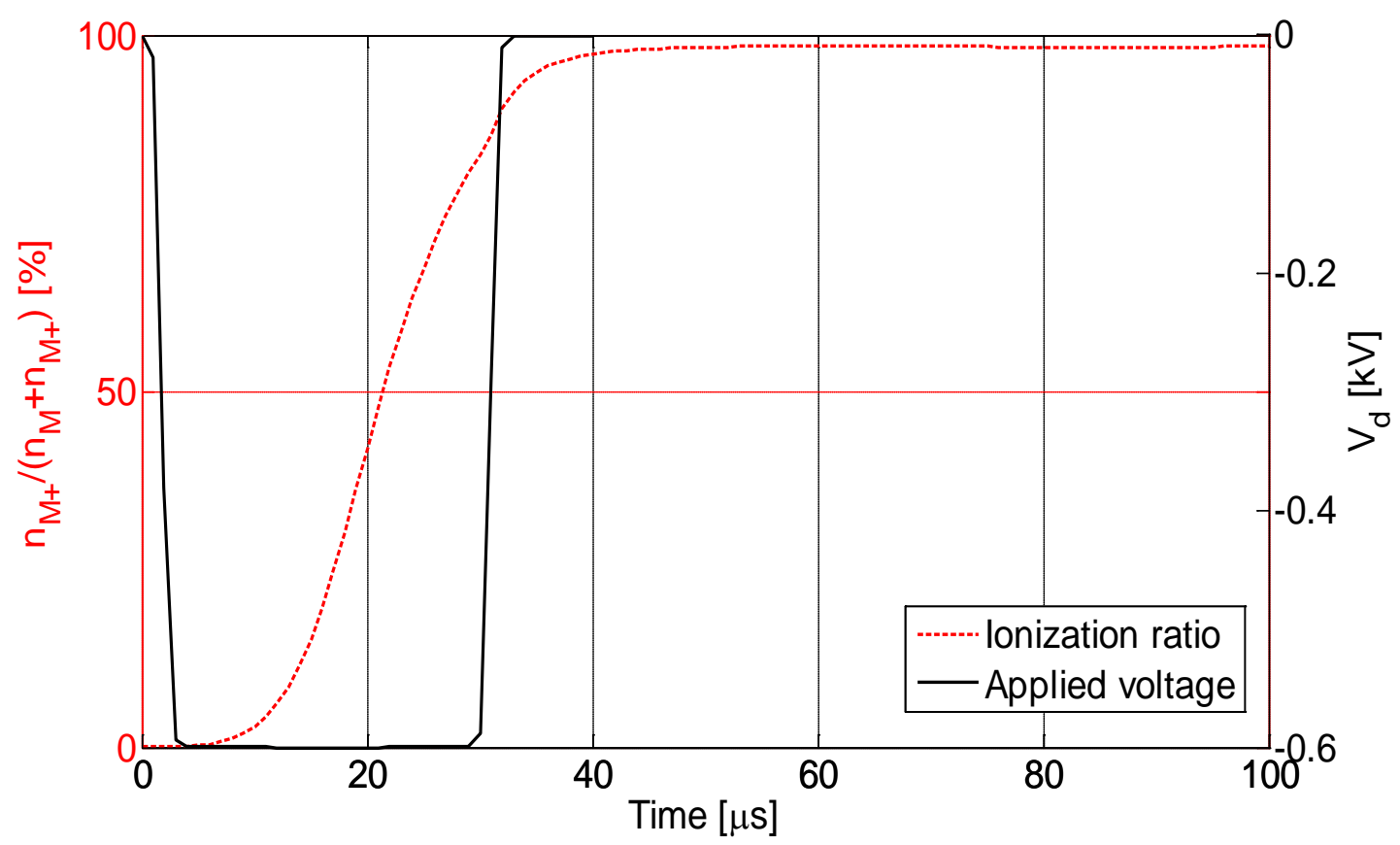

Figure 7: The ionization percentage of sputtered metal ions (dashed red). The applied voltage pulse has been inlaid (solid black) for reference.

\subsection{Extraction of the sputtered species.}

The sputtered species are in the extraction model (sub model 3) treated as minorities that are carried with, and diffuse relative to, the argon gas flow. This assumption can now be motivated by the fact (see figures $6 \mathrm{~b}$ and $6 \mathrm{c}$ ) that both $M$ and $M^{+}$are minorities with densities typically two orders of magnitude below the argon gas density.

Let us first look at the extraction of neutral sputtered atoms through the hollow cathode exit. We define a momentary metal atom extraction efficiency in \% as a function of time as

$$
\eta_{M}(t)=\frac{S_{\text {exit }} \Gamma_{M, \text { exit }}}{S_{H C}\left(\Gamma_{M, H C}+\Gamma_{M^{+}, H C}\right)} \times 100
$$

Here, $S_{\text {exit }}$ is the surface area of the exit, $S_{H C}$ is the inner area of the hollow cathode, and the $\Gamma$ 's denote flux densities for the indicated species and areas. $\Gamma_{M \text {,exit }}$ is obtained from sub model 3, while $\Gamma_{M, H C}$ and $\Gamma_{M^{+}, H C}$ are taken from sub model 2. Equation (1) gives the extraction of metals atoms $M$ as a percentage of the simultaneous total loss of sputtered species to the walls. The result is presented in figure 8 . The curve for metal atoms has a narrow peak of almost $10 \%$ 
efficiency a few microseconds after the pulse is applied, but then decreases and drops below $2 \%$ at the end of the pulse. One reason for this decrease is the growing ionization fraction, as shown in figure 7, which reduces the numerator but not the denominator in Eq. (1). After around $60 \mu$ s, the atom extraction efficiency actually becomes negative. Here, metal atoms are returning into the hollow cathode, against the gas flow direction, because the density gradient of the metal atoms has reversed sign and is so large that the diffusive flux inwards dominates over the convective flux outwards. The density is, however, so low that this particle loss is negligible.

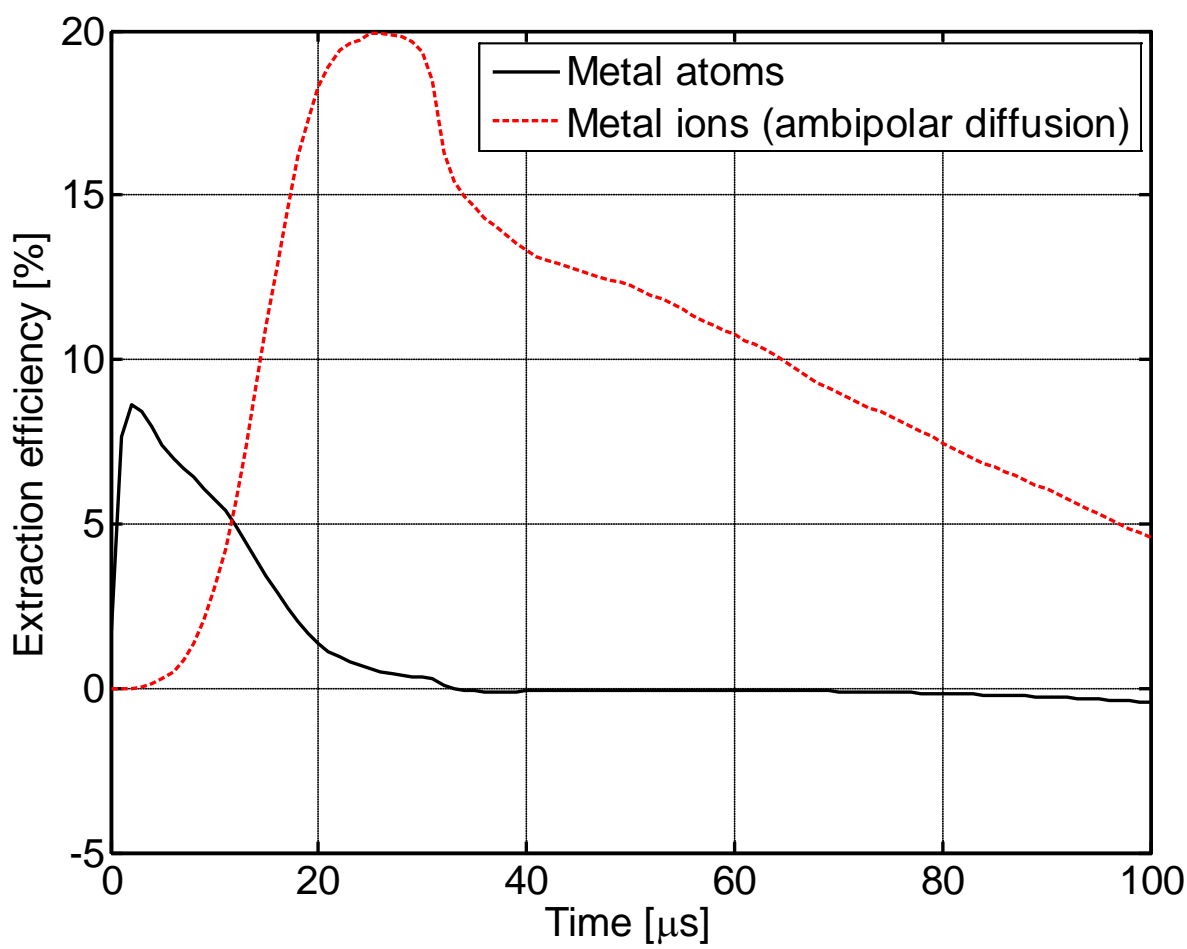

Figure 8: The momentary extraction efficiencies of metal atoms (the solid black line, from equation (1)), and of metal ions (the dashed red line, from equation (2)).

For the metal ions, a momentary extraction efficiency is defined in analogy with Eq. (1) as

$$
\eta_{M^{+}}(t)=\frac{S_{e x i t} \Gamma_{M^{+}, \text {exit }}}{S_{H C}\left(\Gamma_{M, H C}+\Gamma_{M^{+}, H C}\right)} \times 100
$$

It is also shown in figure 8 . It reaches $20 \%$ during the pulse, and continues to be positive and high also after the pulse. This more efficient extraction is because the ambipolar ion diffusion coefficient $D_{a}$ is larger than the diffusion coefficient $D_{n}$ for the neutral species, by a factor of 
$D_{a} / D_{n} \approx \sqrt{T_{e} / T_{n}}$. Taking the temperatures obtained from the modeling (see figure 6), this factor is in the range 3-9 in the pulse. The ambipolar ion extraction therefore is more efficient than the classical diffusion of neutrals. Finally we define average extraction efficiencies, in analogy to the momentary values $\eta_{M}(t)$ and $\eta_{M^{+}}(t)$, as the integrals of the extracted number of particles from the start of the pulse to $100 \mu$ s, divided by the total number $\left(M+M^{+}\right)$lost to the hollow cathode during the same time. For the neutral sputtered species this average extraction efficiency is $3 \%$ and for the metal ions $17 \%$, giving a total extraction efficiency of $17 \%$ (ions) $+3 \%$ (neutrals) $=$ $20 \%$.

The ambipolar diffusion assumption above is most crucial at the exit, where the externally applied field is directed so as to drag ions back into the hollow cathode. At this location we can however directly motivate it as follows. Ambipolar diffusion obtains when the current is zero. In this case the current, expressed in the form of the generalized Ohm's law [8], contains two equally big and opposing terms: one representing the electric conduction and one giving the pressure gradient driven electron flux,

$$
\mathbf{J}=\sigma \mathbf{E}+\frac{\sigma}{q_{e} n_{e}} \nabla P_{e}=0
$$

Here $\sigma$ is the conductivity, $q_{e}$ is the electron charge, $n_{e}$ is the electron density, and $P_{e}$ is the electron pressure. When $J=0, \mathbf{E}$ is the ambipolar electric field $\mathbf{E}_{a p}$. It is directed so as to keep back the electrons and drag the ions in the direction of decreasing pressure. At the exit of the hollow cathode this direction is during the pulse outwards, opposite to the direction of the externally applied field. The pressure gradient term in equation (3) can be estimated from the extraction sub model. During the pulse it is typically one to two orders of magnitude larger than the discharge current density (here approximated by the average over the exit area, $J=I_{D} / S_{\text {exit }}$ ). Thus, $\left|\left(\sigma / q_{e} n_{e}\right) \nabla P_{e}\right|>>|J|$ and, from equation (3), we have approximately the ambipolar diffusion case: $\left(\sigma / q_{e} n_{e}\right) \nabla P_{e}=J-\sigma \mathbf{E} \approx-\sigma \mathbf{E}_{a p}$. It would strictly obtain if $J$ were exactly zero. At the exit of the hollow cathode this means that the electric field reverses its direction compared to the externally applied. In physical terms: the electron pressure gradient at the exit maintains an outwards directed electric field that efficiently extracts ions that are close to the exit. 
The high extraction efficiency of metal ions can thus be attributed to two effects: the electric field reversal during the pulse and, after the pulse, the extra outwards gas flow due to gas heating (see figure 4) that counteracts back diffusion when the density gradient at the exit changes sign.

\section{Experimental verification}

The ideal verification of the complete model would be through direct measurements of the ionization ratio and of the extraction efficiency of the sputtered material. At this stage this is not possible but some indirect verification of the separate sub models can be made by other means. The first lies in the fitting procedure of the experimental current to the modeled current. The plasma chemical sub model is completely locked by the geometry, the initial pressure, the gas and cathode species, and the data set for their interaction. For the fitting of the model to an experimental current there is the only free parameter, the electron heating efficiency fraction, which for energy conservation reasons has to lie in the range $0 \leq F_{P W R} \leq 1$. Furthermore, since most of the energy is most likely dissipated to the ions in the cathode sheath, only a minority of the total dissipated energy should go to the electrons. This further restricts the realistic range to 0 $\leq F_{P W R} \leq 0.5$. The fact that the good agreement during the pulse shown in figure 2 (b) is obtained with a value of $F_{P W R}=0.25$ indicates that the plasma chemical model is based on sound physical assumptions.

For the gas flow and extraction sub models, our means of verification is by comparing the light glow, captured by a CMOS camera, to the computed clouds of hot gas and sputtered species. Figure 9 (a) shows a false-color image taken at an angle to the cathode opening, about $145 \mu$ s from the start of the pulse. The color coding corresponds to the light intensity from the plasma glow (the real color is the characteristic green of copper). The picture was captured for a discharge with a slightly lower flow rate than that used in the model (approximately $60 \mathrm{sccm}$ ), using a CMOS camera (USB uEye SE) that has a maximum frame rate of 87 frames per seconds and a minimum shutter time of 80 micro seconds. The camera was placed outside the vacuum chamber at an angle of 60 degrees to the horizontal plane. A time sequence of the pulsed discharge is obtained by having a mismatch between the frame rate of the camera and the frequency of the discharge. For this purpose, the camera was operated at 20 frames per second, and the discharge at 501 Hz. A light emitting cloud is seen to travel away from the hollow cathode and diffuse. 
Figures 9b and 9c show quite good agreement with the locations, and the transverse extents, of the modeled metal and atom and ion densities.

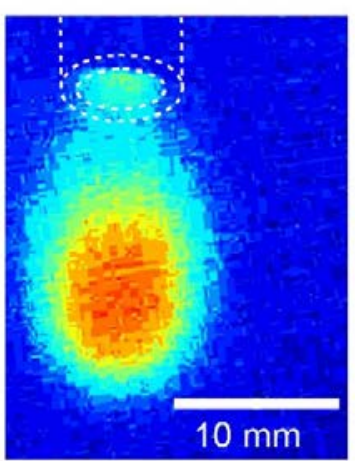

(a)

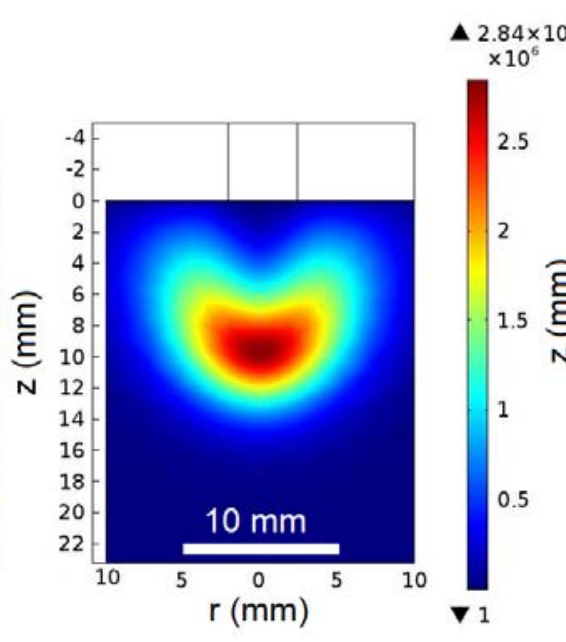

(b)

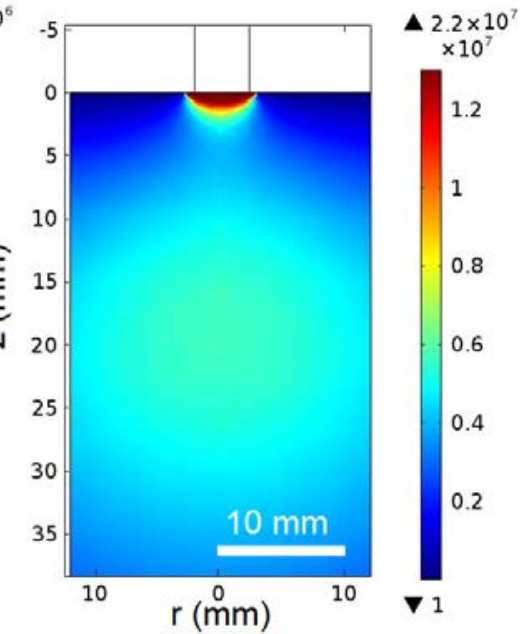

(c)

Figure 9 (a) A false-color image showing the light intensity of the ejected plasma around $145 \mu$ s after the start of the pulse. Notice that the cloud might be artificially elongated due to the $80 \mu$ s shutter time. (b) The metal atom density (arbitrary units). (c) The metal ion density (arbitrary units). The $10 \mathrm{~mm}$ bar in panels (b) and (c) is valid only on horizontal scales.

\section{Summary}

A model is described that follows the discharge and the sputtering process in high power impulse hollow cathode sputtering (HiPICHS), and also the subsequent extraction of material out of the exit of the hollow cathode. The model is built to relate internal parameters, which are usually not directly accessible by measurements, to external input parameters such as the hollow cathode geometry and material, the feed gas flow, the pulse frequency, and the pulse current and voltage curves. The use of the model is exemplified by a special case where the goal is the synthesis of nanoparticles in an expansion volume outside the hollow cathode itself. The desired outputs of the model are here the ionization ratio of the sputtered species, the extraction efficiency, and the time evolutions of the densities and the temperatures of the different species. The ionization ratio obtained for the case under study exceeds $85 \%$, and the extraction efficiencies of sputtered materials are found to be $3 \%$ and $17 \%$ for metal atoms and ions, respectively. The model also 
reveals several physical processes of general interest for pulsed hollow cathode discharges: (1) There can be a significant increase in the extraction efficiency of ions as compared to neutrals due to a plasma-pressure-driven reversal of the electric field at the opening of the hollow cathode during the pulse. (2) Sputtered material is also extracted by an outwards-directed puff of process gas which continues long after the pulse. (3) Back-flow of sputtered material into the hollow cathode after the pulse can be minimized by appropriate combinations of pulse length and feed gas flow. (4) Metastable argon atoms, present in the gas after the pulse, act as an energy reservoir which through superelastic collisions significantly slows down further electron cooling below $T_{e} \approx 1 \mathrm{eV}$. (5) Outside the hollow cathode the extracted neutral atom cloud diffuses through the process gas by classical diffusion, while the ion cloud expands much faster through ambipolar diffusion.

In summary, our case study demonstrates this type of modeling to be a useful tool to relate external discharge data and experimental parameters to internal features of interest such as:

- The sputtered material density inside the hollow cathode, its temperature, and its degree of ionization.

- The efficiencies of extraction of neutral and ionized sputtered material thought the hollow cathode exit.

- The spatial and temporal evolutions outside the hollow cathode of the temperatures and densities of the process gas, the sputtered species, and the plasma electrons.

Such studies have already been central [12] for the physically understanding of how the size of nanoparticles, grown in the range $10-40 \mathrm{~nm}$, depends on pulse power, pulse frequency, and duty cycle. In a continuation of that project the focus is changed to modeling an observed increase in nanoparticle growth rate when the electron temperature in the expansion zone is increased. The model has also been used in a separate project on thin film deposition [10] by pulsed hollow cathodes.

\section{Acknowledgements.}

The author M.I.S would like to thank the European commission for providing him a full scholarship covering his expenses during his study under the Erasmus Mundus external cooperation window scheme and author IP for support from the Swedish Research Council under grant 2008-6572, Linköping Linneaus Environment LiLi-NFM. 


\section{Appendix A. The plasma chemistry sub model}

In this appendix the differences between the IRM [15] and the plasma chemistry sub model 2 are discussed in more details; also modifications of the IRM to accommodate for the new conditions are presented.

A main assumption made in constructing the IRM was that the plasma volume is split into three different regions: sheath region, ionization region (where the majority of ionization reactions occur) and bulk plasma region. The IRM and the plasma chemistry model presented here are defined in the ionization regions. To derive the plasma chemistry model from the IRM, certain modifications have to be done to account for differences in the physical description between the magnetron case and the hollow cathode case. There are two main differences between these two cases. First, the hollow cathode discharge is collision dominated while the IRM model is a collision free model. Second, see Fig. 1, the difference in the surface area of the ionization region facing the bulk plasma volume between the two cases is large. In the IRM almost $70 \%$ of the surface of ionization region faces the bulk plasma while in the hollow cathode discharge that percentage is less than $10 \%$.

To account for those differences three major modifications have been done in the plasma chemistry model compared to the IRM. The first modification applies to the density correction factor, which is a dimensionless geometrical factor relating the averaged densities to the densities at the ionization-region/sheath interface. The new factor for cylindrical geometries is given by [16]:

$$
h_{R}=0.8\left[4+\frac{R}{\lambda_{i}}+\left(\frac{0.8 R u_{B}}{\chi_{01} J_{1}\left(\chi_{01}\right) D_{a}}\right)^{2}\right]^{-0.5}
$$

The factor given by equation (A.1) is used for the charged species, while the factor used for neutral species is $h_{R}=0.25$. $R$ is the radius, $\lambda_{i}$ the mean free path of ions, $u_{B}$ the Bohm velocity of ions, $D_{a}$ the ambipolar diffusion coefficient, $J_{1}$ the first order Bessel function, and $\chi_{01}$ the first zero of the zero order Bessel function.

The second modification applies to the argon atom density equation (the continuity equation). In the IRM, the recombined $\mathrm{Ar}^{+}$ions (i.e., argon atoms) returning from the cathode were approximated as a loss. However, in the plasma chemistry model the majority of the argon ions 
lost to the walls will recombine and stay in the hollow cathode volume, thus they represent a gain term for the argon atom equation. The new term is the right most term in equation (A.2); for comparison/reference the reader is referred to equation (4) of [15]:

$$
\frac{d n_{A r}}{d t}=-k_{i z} n_{e} n_{A r}-k_{e x c} n_{e} n_{M}+k_{d e x} n_{e} n_{A r^{m}}+k_{p} n_{M} n_{A r^{m}}+k_{\text {chexc }} n_{M} n_{A r^{+}}+\frac{\Gamma_{A r^{+}} S_{H C}}{V_{I R}}
$$

In equation (A.2) $n_{i}$ denotes the density of electrons $(i=e)$, argon atoms ( $\left.i=A r\right)$, argon ions $\left(i=A r^{+}\right)$, metastable argon atoms $\left(i=A r^{m}\right), S_{H C}$ is the side surface area of the hollow cathode and $V_{I R}$ is the volume of the ionization region; $\Gamma_{\mathrm{Ar}}$ is the flux of argon ions to the walls of the hollow cathode, and $k$ denotes the rate coefficient of the reactions given in literature: ionization (iz)[17], excitation (ex)[18], de-excitation (dex)[13], penning ionization $(p)$ [19], and charge exchange ionization (chexc)[19]. The third modification is the construction of a new energy equation for the heavy species to replace a kinetic treatment used in the IRM. Since the experimental discharge of investigation is collision dominated, we assume that all the heavy species have the same temperature $\left(T_{\text {heavy }}\right)$. The equation for the temperature of all heavy species has been derived from heat equation for ideal gases [20]:

$$
\begin{aligned}
\frac{3}{2} \sum_{\alpha=1}^{5} n_{\alpha} \frac{d T_{\text {heavy }}}{d t} & =E_{\text {el,Ar }} k_{e l, A r} n_{e} n_{A r}+E_{\text {el, } M} k_{\text {el, } M} n_{e} n_{M}+\frac{w_{M, A r} Y_{\text {sput }} \Gamma_{A r} S_{H C}}{V_{I R}}+\frac{w_{M, M} Y_{\text {self }} \Gamma_{M^{+}} S_{H C}}{V_{I R}}+\frac{w_{A r, r e c} \Gamma_{A r} S_{H C}}{V_{I R}} \\
& -\frac{3}{2} \frac{\left(T_{\text {heavy }}-T_{\text {wall }}\right) \Gamma_{A r} S_{H C}}{V_{I R}}-\frac{3}{2} T_{\text {heavy }} \frac{d \sum_{\alpha=1}^{5} n_{\alpha}}{d t}
\end{aligned}
$$

In equation (A.3), $E_{e l, A r}$ and $E_{e l, M}$ are the energies gained by the heavy species due to elastic collisions between electrons and argon atoms and electrons and metal atoms respectively, $\Gamma_{M^{+}}$is the metal ions flux to the walls, $Y_{\text {sput }}$ and $Y_{\text {self }}$ are the sputtering yields of metal by argon and metal ion bombardment respectively, and $T_{\text {wall }}$ is the temperature of the wall of the hollow cathode. On the right hand side of equation (A.3), the first two terms describe the heating due to elastic collisions with electrons. The third term describes the heat flux due to sputtered metal atoms by metal ions bombardment. The $4^{\text {th }}$ term is the heat flux due to sputtered metal atoms by argon ions bombardment, and the $5^{\text {th }}$ term is the heat flux due to recombined argon ions. The $6^{\text {th }}$ term is the heat flux due to energy exchange with the wall. The averaged energy carried per sputtered atom ( $w_{M, M}$ and $\left.w_{M, A r}\right)$ was computed using a modified Thompson formula [21]. The average energy of the recombined argon ion returning from the walls has been set to $2 \mathrm{eV}$ [15]. 
The equation for the electron temperature is given in Equation A.4. The pendulum electrons effect was neglected in this formulation for simplicity. Equation A.4 has the same terms as the IRM [15] has. For that reason no further discussion of the formulation will be presented here:

$$
\begin{aligned}
& \frac{3}{2} n_{e} \frac{d T_{e}}{d t}=\frac{F_{p w r} I_{D} V_{D}}{q_{e} V_{I R}}+E_{e x c} k_{d e x} n_{e} n_{A r^{m}}-\left(E_{i z}+\frac{3}{2} T_{e}\right) k_{i z} n_{e} n_{A r}-\left(E_{i z m}+\frac{3}{2} T_{e}\right) k_{i z m} n_{e} n_{M}-\frac{T_{e}}{2} \frac{\left(\Gamma_{A r^{+}}+\Gamma_{M^{+}}\right) S_{H C}}{V_{I R}} \\
& -\left(E_{\text {exciz }}+\frac{3}{2} T_{e}\right) k_{e x c i z} n_{e} n_{A r^{m}}-\left(E_{e x c}-E_{i z m}+\frac{3}{2} T_{e}\right) k_{P} n_{M} n_{A r^{m}}-E_{e l, A r} k_{e l, A r} n_{e} n_{A r}-E_{e l, M} k_{e l, M} n_{e} n_{M}
\end{aligned}
$$

In Equation (A.4), $q_{e}$ is the electron charge, $T_{e}$ is the electron temperature. All "E"s are reaction energies: excitation of argon atoms to metastable level $\left(E_{\text {exc }}\right)$, argon ionization $\left(E_{i z}\right)$, metal ionization $\left(E_{\text {izm }}\right)$, ionization from metastable level $\left(E_{\text {exciz }}\right) . k_{\text {izm }}$ is the metal ionization reaction coefficient, $k_{\text {exciz }}$ is the metastable ionization reaction coefficient,,$I_{D}$ and $V_{D}$ are the experimental discharge current and voltage respectively. $F_{p w r}$ is the dimensionless fitting parameter; the term it appears in represents the energy source for the chemical reactions induced by the electrons. The obtained value of $F_{p w r}$ for this run was 0.25 . Comparing the "effective" power portion delivered to electrons (given by $F_{p w r} I_{D} V_{D}$ ) to the power delivered to electrons by accelerating secondary emitted electrons in the sheath (estimated by $\gamma_{s e c} I_{D} V_{D}$ ) where $\gamma_{\text {sec }}$ is the secondary electrons yield, we find that the effective power is larger as $F_{p w r}$ is 0.25 while $\gamma_{s e c}$ is 0.1. This relatively high value of $F_{p w r}$ might be attributed to a more efficient ionization in the sheath in the hollow cathode geometry compared to HiPIMS [22], and perhaps pendulum electrons.

Finally, the modeled current is given by:

$$
I_{D}(t)=\left(\left(1+\gamma_{M}\right) \times \Gamma_{M^{+}}+\left(1+\gamma_{A r}\right) \times \Gamma_{A r^{+}}\right) q_{e} S_{H C}
$$

where $\gamma_{M}$ and $\gamma_{A r}$ are the secondary emission coefficients due to bombardment by metal and argon atoms respectively. Equation A.5 is only valid during the pulse-on time because it assumes that the electron flux at the cathode's surface is given by the secondary electron emission flux. As soon as the pulse is switched off, the electrons flux from the ionization region toward the cathode increases rapidly causing the discharge current to drop immediately to zero. Computing the electron flux to the walls is outside the scope of this study. 
The plasma chemistry sub model, consisting of 8 ordinary differential equations (5 continuity equations for argon atoms, argon ions, metastable argon, metal atoms and metal ions, two energy equations for electrons and heavy species, and one equation for computing $F_{p w r}$ ) were solved using a MATLAB built in stiff solver (Runge-Kutta based solver). The electron density at every time was calculated from quasi neutrality by adding the densities of argon ions and metal ions.

\section{Appendix B. COMSOL sub models}

In this work there were two COMSOL sub models which are the gas flow model and the extraction model. Starting by the flow sub model, it is a 2D time dependent model defined everywhere in the computational domain (see figure 4a). It solves fluid flow equations for argon gas (assumed to be an ideal gas), the system of equation solved is:

$$
\begin{gathered}
\frac{\partial \rho}{\partial t}+\nabla \cdot(\rho \vec{v})=0 \\
\rho\left(\frac{\partial \vec{v}}{\partial t}+(\vec{v} \cdot \nabla) \vec{v}\right)=-\nabla P+\mu \nabla^{2} \vec{v}+\frac{1}{3} \mu \nabla(\nabla \cdot \vec{v}) \\
\rho C_{p}\left(\frac{\partial T}{\partial t}+(\vec{v} \cdot \nabla) T\right)=\nabla \cdot(\kappa \nabla T)+Q
\end{gathered}
$$

Equation B.1 is the continuity equation for argon gas, $\rho$ is the argon gas density and $v$ is the velocity field. Equation B.2 is the momentum conservation equation, $P$ is the pressure and $\mu$ is the viscosity of argon gas. Equation B.3 is the heat equation, $T$ is the temperature of argon gas. $C_{p}$ is the heat capacity at constant pressure, $\kappa$ is the heat conductivity. $Q$ is a volumetric heat source. The values of $C_{p}, \mu$ and $\kappa$ of argon are taken from COMSOL materials library. The three equations (B.1, B.2 and B.3) were solved using time dependent non-isothermal flow module.

Equations B.1 and B.2 were solved in steady state mode (setting all time derivative terms to zero) to obtain the initial conditions before the pulse. All boundaries are assumed to be walls (i.e $v=0$ ) except the inlet at $\mathrm{z}=50 \mathrm{~mm}$ where a constant normal velocity of $26 \mathrm{~ms}^{-1}$ in negative $\mathrm{z}$ direction is defined, this particular value was chosen to fulfill the requirement of having a flow rate of 100 sccm along the axis of the hollow cathode. A gas outlet is defined at $\mathrm{z}=-120 \mathrm{~mm}$ by a constant pressure of $133 \mathrm{~Pa}$. Please refer to figure 3a.

For the pulsed operation, the same boundary conditions as in the steady state case are used. The difference is that equation B.3 is now solved in addition to B.1 and B.2. The three equations are solved as function of time. The boundary conditions for equation B.3 are thermal insulators (i.e normal heat flux is set to zero) except at the cathode wall where a heat flux is defined as follow: 


$$
q_{\text {heat }}=q_{e}\left(w_{M, M} Y_{\text {self }} \Gamma_{M^{+}}+w_{M, A r} Y_{\text {sput }} \Gamma_{A r^{+}}-\frac{1}{2}\left(T-T_{\text {wall }}\right) \Gamma_{A r}\right)
$$

In equation B.4 there are three terms, the first is the heat flux due to sputtered metal atoms by metal ions, the second term is the heat flux due to sputtered metal atoms by argon ions, and the third term is the hot argon atoms flux ( with temperature $T$ ) exchanging heat with wall ( with temperature $T_{\text {wall }}=293.5 \mathrm{~K}$ ). The heat flux given in equation B.4 is a function of $T$ (argon gas temperature). All the other parameters are given by the plasma chemistry sub model. In addition to the heat flux from the cathode wall, there is a volumetric heat source term defined only in V1 (see figure 3a). It is defined by:

$$
Q=3 q_{e} m_{e} n_{e} T_{e}\left(\frac{k_{e l, M} n_{M}}{m_{M}}+\frac{k_{e l, A r} n_{A r}}{m_{A r}}\right)
$$

Equation B.5 consists of two terms representing energy gained from electrons by elastic collisions with metal atoms and argon atoms respectively. The initial condition of equation B.3 is assumed to be a constant temperature everywhere that is equal to $293.5 \mathrm{~K}$. The initial conditions of equations B.1 and B.2 are the solutions from the steady state run.

The extraction sub model is a 2D time dependent model that is only defined in V2 as depicted in figure 3a. For the metal atoms density the equation solved is:

$$
\frac{\partial n_{M}}{\partial t}+\nabla \cdot\left(-D \nabla n_{M}+\vec{v} n_{M}\right)=0
$$

In equation B.6,D is the diffusion coefficient of metal atoms. It is a function of temperature which is taken from the flow model. $v$ is the convection velocity which is the flow velocity of argon gas taken from gas flow model as well. The boundary condition defined over all the boundaries except the common surface between V1 and V2 (see figure 3a) is outward thermal flux (i.e. $\Gamma_{\text {thermal }}=-n_{M} v_{t h} / 4$ ). The common surface between V1 and V2 is an inlet defined by time dependent density obtained from chemistry sub model. Equation B.6 was solved using time dependent PDE coefficient form in COMSOL.

For the metal ions density, since ambipolar diffusion assumes quasi neutrality condition holds, the drift diffusion equations were solved for electrons. Two equations were solved one for electron density and one for electron temperature:

$$
\begin{aligned}
& \frac{\partial n_{e}}{\partial t}+\nabla \cdot\left(-D_{a} \nabla n_{e}\right)+\vec{v} \cdot \nabla n_{e}=0 \\
& \frac{\partial n_{\varepsilon}}{\partial t}+\nabla \cdot\left(-D_{a} \nabla n_{\varepsilon}\right)+\vec{v} \cdot \nabla n_{\varepsilon}=0
\end{aligned}
$$


In equations B.7 and B.8, $D_{a}$ is the ambipolar diffusion coefficient, $v$ is the convection velocity taken from flow sub model, and $n_{\varepsilon}$ is the electron energy density. The convection velocity affects the electrons indirectly by affecting the ions which affect the electrons through ambipolar electric field. Equation B.8 is solved for the electron energy density from which the electron temperature is obtained. For the derivation of this formulation see [23]. Equations B.7 and B.8 were solved using the drift diffusion module.

The boundary condition for Equations B.7 and B.8 defined over all the boundaries except the common surface between V1 and V2 (see figure 3a) is outward thermal flux ( $\Gamma_{e_{-} \text {thermal }}=$ $n_{e} v_{e_{-} t h} / 2$ and $\left.\Gamma_{\varepsilon_{-} \text {thermal }}=-5 n_{\varepsilon} v_{e_{-} t h} / 6\right)$. The common surface between V1 and V2 is an inlet defined by time dependent electron density and temperature obtained from chemistry sub model. To obtain the density and flux of metal ions density after the model is solved; the electron density is multiplied by the ratio of metal ions to total ions. This ratio is obtained from plasma chemistry model.

\section{References:}

[1] Ferreira C M and Delcroix J L 1978 J. Appl. Phys. 492380

[2] Kennedy R V 2001 J. Phys. D: Appl. Phys. 34787

[3] Paduraru C, Becker K H, Belkind A, Lopez J L and Gonzalvo Y A 2007 IEEE Transactions on Plasma Science 35527

[4] Fearn D G and Peterson S W 2000 ESA special publications 465587

[5] Kouznetsov V, Macák K, Schneider J M, Helmersson U and Petrov I 1999 Surf. Coat. Technol. 122290

[6] J. T. Gudmundsson, N. Brenning, D. Lundin, and U. Helmersson 2012 J. Vac.Sci. Technol. A 30030801

[7] Lidsky L M, Rothleder S D, Rose D J, Yoshikawa S, Michelson C and Mackin R J 1962 J. Appl. Phys. 332490

[8] Chen F 1984 Introduction to plasma physics and controlled fusion $2^{\text {nd }}$ edition Springer p 185

[9] Raizer Y P 1991 Gas Discarge Physics Berlin Springer p 31 
[10] Pedersen H., Larsson P., Aijaz A., Jensen J. and Lundin D. 2012 Surface \& Coatings Technology 2064562

[11] Helmersson U, Brenning N and Söderström D 2012 Swedish Patent SE 535381

[12] Pilch I, Söderström D, Brenning N, and Helmersson U. Appl. Phys. Lett. 2013102033108

[13] Ashida S, Lee C and Lieberman M A 1995 J. Vac. Sci. Technol. A 132498

[14] Gudmundsson J T 2008 J. of physics conference series 10008201311

[15] Raadu M A, Axnäs I, Gudmundsson J T, Huo C and Brenning N 2011 Plasma Sources Science and Technology, 20065007

[16] Lieberman M A and Lichtenberg A J 2005 Principles of plasma discharge and materials processing $2^{\text {nd }}$ edition Wiley p 149

[17] Gudmundsson J T and Thorsteinsson E G 2007 Plasma Sources Sci. Technol. 16399

[18] Lee M H and Chung C W 2005 Phys. Plasmas 12073501

[19] Lu J and Kushner M J 2000 J. Appl. Phys. 877198

[20] Physics of Continuous Matter, Exotic and Everyday Phenomena in the Macroscopic World, B Lautrup, chapter 30.

[21] Stepanova M and Dew S K 2004 Nuclear Instruments and Methods in Physics Research B 215 357-365

[22] Depla D, Mahieu S and De Gryse R 2009 Thin solid films 517 2825-2839

[23] Hagelaar G and Pitchford L 2005 Plasma Sources Sci. Technol. 14722 